conversion seizures. Attacks were characterized by auditory hallucinations, vertigo, automatisms, screaming, and impaired consciousness. A simple partial seizure progressed to a complex partial seizure and then evolved into a NES. She had a history of sexual abuse. MRI revealed right frontoparietal dysplasia, an area critical for impulse control and behavioral inhibition. The epileptic seizure may trigger the conversion NES, especially in a patient with right frontal lobe pathology.

\title{
EMERGENCY CT IN NEW ONSET SEIZURES
}

A retrospective analysis of 107 neurologically normal children with emergency brain scans for new-onset seizures is reported from the Children's National Medical Center, Washington, DC. In $8(7 \%)$, the seizures were nonepileptic (gastroesophageal reflux, syncope, rigor). In 49 of the remaining 99 , seizures were provoked (complicated febrile seizure, encephalitis, toxic or metabolic), and in 50, they were unprovoked. CT abnormalities identified in 19 required further investigation or intervention in 7 (tumors in 2, vascular anomalies in 3 , cysticercosis in 1 , and obstructive hydrocephalus in 1). First seizures unprovoked by fever or metabolic causes, and especially focal seizures, were most likely to have underlying pathologies demonstrated on emergency CT and requiring intervention. (Garvey MA, Gaillard WD, Rusin JA et al. Emergency brain computed tomography in children with seizures: who is most likely to benefit? I Pediatr Nov 1998;133:664-669). (Reprints: WD Gaillard MD, Department of Neurology, Children's National Medical Center, 111 Michigan Ave, NW, Washington, DC 20010).

COMMENT. Emergency CT is indicated in a previously well child with a newonset unprovoked seizure, especially if the seizure is focal. A first seizure with fever is unlikely to be symptomatic of an unexpected brain lesion demonstrable by CT and requiring intervention. A careful neurologic exam performed within 24 hours of the seizure, to exclude localized abnormalities and asymmetry of signs, is essential in determining the need for CT. When the history is unreliable, the neurologic signs of uncertain significance, and follow-up unavailable, a CT is advisable before the patient is discharged.

\section{EPILEPSY SURGERY OUTCOME}

Seizure outcome was studied in 136 children receiving surgery for intractable epilepsy at the Cleveland Clinic between 1990 and 1996. The postoperative follow-up period was 1 to 7.5 years (mean, 3.6 years). Cortical dysplasia and low-grade tumor were the most common causes; hippocampal sclerosis was rare. A seizure-free outcome achieved in $60-69 \%$ of patients was more frequent in patients requiring temporal resections (74-80\%) than in those with extratemporal or multilobar resection (52-58\%), and also among patients with tumor (82\%) compared to those with cortical dysplasia (52\%). Age was not a factor; the frequency of a seizure-free outcome was similar for infants, children, and adolescents, and comparable to reports of adult series. (Wyllie E, Comair YG, Kotagal $\mathrm{P}$ et al. Seizure outcome after epilepsy surgery in children and adolescents. Ann Neurol Nov 1998;44:740-748). (Respond: Dr Wyllie, Pediatric Epilepsy Program, The Cleveland Clinic Foundation, 9500 Euclid Ave, Cleveland, OH 44195).

COMMENT. The risk/benefit ratio for surgery of intractable epilepsy varies with the pathology and localization of the lesion. Patients with low-grade tumors and foci located in the temporal lobe have a better outcome than those with cortical dysplasia and extratemporal or hemispheric localization. Outcome is not 